\title{
THE SECOND GUIDE STAR CATALOGUE
}

\author{
B. McLEAN ${ }^{1}$, G. HAWKINS ${ }^{1}$, A. SPAGNA $^{2}$, M. LATTANZI ${ }^{2}$, \\ B. LASKER ${ }^{1}$, H. JENKNER ${ }^{1,3}$ AND R. WHITE ${ }^{1}$ \\ ${ }^{1}$ Space Telescope Science Institute \\ ${ }^{2}$ Osservatorio Astronomico di Torino \\ ${ }^{3}$ European Space Agency
}

\section{Introduction}

Although the HST GSC-I (Paper-I: Lasker et al. 1990, Paper-II: Russell et al. 1990, Paper-III: Jenkner et al. 1990) has been used with great success operationally, it was always known that it was possible to improve the scientific and operational usefulness by an increase in scope to include multi-color and multi-epoch data. Once the GSC-II concept was established, it was evident that, even beyond the original motivations in HST operations, it would address a number of other astronomical needs such as increasing demands for fainter catalogues to support remote or queue scheduling capabilities and adaptive optics on the next generation of largeaperture, new-technology telescopes. In addition, the all sky nature of the GSC-II makes it a natural data source for research in galactic structure.

\section{The GSC-II Project}

Once the broad applications of the GSC-II were recognized and its resource requirements were considered with care, it was clear that the project needed to be expanded beyond its original ST ScI context. This led to the formation of a GSC-II consortium (STScI, the Italian Council for Research in Astronomy and the Astrophysics Division of the European Space Agency), with the formal goal of producing the GSC-II as an all-sky catalogue of positions, proper motions, magnitudes and colours, complete to 18 th magnitude. 


\section{GSC-II Overview}

The GSC-II will be constructed using the digitized sky survey plates available at STScI. The POSS-II (J and F plates), ER, and SES surveys have finer grain emulsions, hence better resolution, and a deeper limiting magnitude than the original POSS surveys while the first epoch plates can be used to provide a baseline to determine the stellar proper motions.

The Guide Star Photometric Catalog (GSPC-I; Lasker et al. 1988) is being supplemented by CCD observations to provide a set of standard stars to at least 18th magnitude over the entire sky for each Schmidt plate. The future availability of the HIPPARCOS and TYCHO catalogues will provide a dense grid of very accurate astrometric standards to tie the plates to the inertial reference frame and may allow the mapping of field variations in the sensitivity of the plate emulsions. The addition of texture values for each object and the ranking of all object parameters has proven to be an effective technique for training a decision tree classifier and providing more reliable object classifications.

The improvement in the projected precision of GSC-II compared to the original GSC based on the current prototype software is shown in Table 1.

TABLE 1. Properties of GSC-I and GSC-II

\begin{tabular}{lll}
\hline Property & GSC 1.1 & GSC 2.0 \\
\hline Number Objects & $1.8 \times 10^{7}$ & $2.0 \times 10^{9}$ \\
Epochs & 1 & $2-3$ \\
Passbands & 1 & $>2$ \\
Limiting Magnitude & 15 & $>18$ \\
Relative/Absolute Position Error & $0.4^{\prime \prime} / 1.0^{\prime \prime}$ & $0.15^{\prime \prime} / 0.5^{\prime \prime}$ \\
Proper Motion Error & $\mathrm{n} / \mathrm{a}$ & $<4.0 \mathrm{mas} \mathrm{yr}^{-1}$ \\
Magnitude Error & $0.4 \mathrm{mag}$ & $0.1-0.2 \mathrm{mag}$ \\
Classification Accuracy & $99 \%$ stellar & $>95 \%$ unbiased \\
\hline
\end{tabular}

\section{References}

Jenkner, H., et al. 1990 Astron. J. 99, 2081

Lasker, B. M., et al. 1988 Astrophys. J. Suppl. 68, 1

Lasker, B. M., et al. 1990 Astron. J. 99, 2019

Lasker, B. M. 1994, Proceedings of Astronomy from Wide Field Imaging, IAU Symp. 161, p. 235, MacGillivray et al. (eds)

Russell, J. L., et al. 1990 Astron. J. 99, 2059 